Published in final edited form as:

J Contin Educ Health Prof. 2020 ; 40(4): 235-241. doi:10.1097/CEH.0000000000000305.

\title{
Development of an Instrument to Assess the Perceived Effectiveness of Academic Detailing
}

\author{
Mary H. Smart, PharmD, MS, \\ Department of Pharmacy Systems, Outcomes and Policy, College of Pharmacy, University of \\ Illinois at Chicago, Chicago, IL \\ Andrea L. Monteiro, MSc, \\ Department of Pharmacy Systems, Outcomes and Policy, College of Pharmacy, University of \\ Illinois at Chicago, Chicago, IL

\section{Christopher D. Saffore, PharmD,} \\ Department of Pharmacy Systems, Outcomes and Policy, College of Pharmacy, University of \\ Illinois at Chicago, Chicago, IL
}

\section{Aleksandrina Ruseva [PharmD Candidate], \\ Department of Pharmacy Systems, Outcomes and Policy, College of Pharmacy, University of Illinois at Chicago, Chicago, IL}

Todd A. Lee, PharmD, PhD, Department of Pharmacy Systems, Outcomes and Policy, College of Pharmacy, University of Illinois at Chicago, Chicago, IL

\section{Michael A. Fischer, MD, MS,}

Division of Pharmacoepidemiology and Pharmacoeconomics, National Resource Center for Academic Detailing, Brigham and Women's Hospital, Boston, MA

\section{Alan Simon Pickard, PhD}

Department of Pharmacy Systems, Outcomes and Policy, College of Pharmacy, University of Illinois at Chicago, Chicago, IL

\section{Abstract}

Introduction: Academic detailing (AD) is an effective, evidence-based education outreach method of promoting clinician behavior change. Detailer feedback is important for program evaluation but is rarely systematically collected. The study's objective was to develop a measure capturing the detailer's perception of the effectiveness of an AD program.

Methods: A six-item measure with a five-level scale was initially developed from the literature review and expert panel consultation. Item constructs were usefulness, acceptability, feasibility, relevance, effectiveness of communication, and readiness to change. The measure was piloted,

Correspondence: A. Simon Pickard, PhD, Department of Pharmacy Systems, Outcomes and Policy, College of Pharmacy, University of Illinois at Chicago, 833 South Wood Street, M/C 886, Chicago, IL 60612; pickard1 @ uic.edu.

Supplemental digital content is available for this article. Direct URL citations appear in the printed text and are provided in the HTML and PDF versions of this article on the journal's Web site (www.jcehp.org). 
refined, and tested during an opioid-focused AD program that included two visits. The instrument structure was evaluated using exploratory factor analysis, measure reliability was assessed using item-item correlation (rho), corrected item-total correlation, Cronbach alpha (a), and item response theory.

Results: The initial six-item instrument demonstrated unidimensionality. The Cronbach a for the measure was 0.74 (visit 1) and 0.79 (visit 2); one item (relevance) was redundant ( $a=0.73$ and 0.79 when deleted) and therefore dropped. Items related to usefulness, acceptability, and readiness to change displayed high item-item correlation (rho 20.50 ) and contributed the most information and seemed to operate as a single scale (ie, "likelihood to change") based on item response theory analysis. Items related to feasibility and communication were slightly different constructs and should be reported separately.

Discussion: The five-item detailer assessment of visit effectiveness (the "DAVE") instrument provides a standardized approach to assess AD. Further study of its validity and broader use in other programs and educational outreach activities is encouraged.

\section{Keywords}

academic detailing; opioid; instrument; psychometrics; educational outreach

The opioid epidemic continues to be a major public health concern in the United States (US). In 2017, the Center for Disease Control and Prevention (CDC) reported that over 35\% of overdose-related deaths were associated with prescription opioids. ${ }^{1}$ Primary care specialties account for most opioid prescriptions dispensed in the United States ${ }^{2,3}$ However, primary care providers (PCPs) struggle to balance the difficulty of managing patients with chronic pain with concern for opioid misuse and addiction among their patients. ${ }^{4}$ Limited medical training in pain management contributes to the lack of confidence in managing patients with chronic pain and serves to highlight the need for adequate continuing education in pain management. ${ }^{5-8}$ Tailored educational strategies, such as academic detailing (AD), can address training deficits in appropriate pain management and modify opioid prescribing behavior.

$\mathrm{AD}$ is an educational outreach approach to provide clinicians with current, evidence-based information to modify clinical decision-making and change practice behavior. ${ }^{9,10} \mathrm{AD}$ is characterized by one-on-one visits with health care providers by specially trained personnel (ie, academic detailers). ${ }^{11}$ Proctor et al ${ }^{12}$ 's Implementation Research Outcomes Model identifies certain implementation outcomes such as feasibility, fidelity, acceptability, and uptake that are critical to consider in the successful implementation of an AD program. Based on the clinician feedback from initial visits, the acceptability and feasibility of the program can be assessed, and future AD visits can be tailored to effectively reinforce key messages while building rapport with the clinician over time. ${ }^{13}$ However, in lieu of direct clinician feedback, capturing the detailer's perception of the acceptability and feasibility of the AD visits may be useful to inform the development, implementation, and evaluation of future AD programs. 
Previous studies have used AD to modify clinician decision-making and influence prescribing behavior related to opioids. ${ }^{14-20}$ However, these studies have lacked the use of a standardized validated tool (eg, postvisit survey or questionnaire) to measure AD visit effectiveness. ${ }^{14,17,18,20}$ Van Hoof et $\mathrm{al}^{21}$ reported that $\mathrm{AD}$ studies have poor and incomplete documentation of $\mathrm{AD}$ visit details, and it is unknown as to the extent to which these details actually affect the $\mathrm{AD}$ visit effectiveness. Although a study reported detailer feedback, it captured the detailer's perception in an unsystematic manner. ${ }^{22}$ Furthermore, no opioidrelated $\mathrm{AD}$ studies have captured the detailer perception. Therefore, we sought to develop and evaluate the psychometric properties of an instrument to assess the detailer's perception of an opioid-focused $\mathrm{AD}$ program which would be generalizable across $\mathrm{AD}$ programs.

\section{METHODS}

The goal of the measure was to assess the perceived effectiveness of the educational outreach visit by the detailer. The purpose of such a measure could serve to support continuous quality improvement of AD programs and includes components that explain or predict the effectiveness of an AD program in various providers. The approach to measure development followed established steps: item generation based on the literature search and expert input, item selection and refinement, formatting, piloting and refinement, and largerscale validation. ${ }^{23}$

\section{Item Generation and Selection}

A structured literature review was conducted using MEDLINE and Google Scholar from inception to April 2018. The search was limited to studies published in English, in peerreviewed journals, and those that reported satisfaction with $\mathrm{AD}$ and educational outreach programs. The search strategy included a combination of the following terms, accompanied by MeSH terms where appropriate: "academic detailing," "educational outreach," "experience," "satisfaction," and "acceptability."

Results of the search strategy were screened, and relevant articles were retrieved by independent reviewers who were trained in the systematic review process and several who had previously conducted and published articles on systematic reviews. Although some of the articles on educational outreach reported a standardized approach to capture prescriber satisfaction with the program, no studies reported a standardized, systematic approach to assess the perspective of the detailer. Based on important elements identified in the literature, relevant themes for item generation included usefulness, acceptability, feasibility, topic relevance, and communication effectiveness. We followed a thematic analysis framework to identify the themes according to the frequency of appearance and relevance to $\mathrm{AD}$ in the identified literature. The items were generated with input from the research team, which included expertise in educational measurement, psychometrics, and survey development. The items and response options structure were then circulated among members of an expert advisory committee on clinical care that included patient advocates and faculty from several academic institutions in Illinois. An additional item related to readiness to change was added, and the wording was refined. There was support for the proposed scale responses based on intensity rather than frequency of response. A Likert-type

J Contin Educ Health Prof. Author manuscript; available in PMC 2021 October 01. 
scale was used to rate the detailer's perception of the intervention ranging from "not at all" = 1 , "slightly" $=2$, "moderately" $=3$, "very" $=4$, and "extremely" $=5$. The content relevance and representativeness of the instrument were then rereviewed by the content experts (ie, physicians and pharmacists with extensive training in pain management), before field testing of the instrument.

\section{Field Testing of the Measure}

The initial measure was field tested by trained academic detailers who participated in an $\mathrm{AD}$ program. The AD program was developed to assess the impact of detailing on opioid prescribing activities among PCPs at a large health system in the greater Chicago area. The AD program comprised two face-to-face visits with PCPs from specially trained detailers from June 2018 to September 2018. Each visit was approximately 15 minutes. Follow-up visits were scheduled approximately 6 to 8 weeks apart. Ten detailers from the College of Pharmacy at the University of Illinois at Chicago, consisting of first-year and second-year Doctor of Pharmacy students and two Doctor of Philosophy students with previous clinical pharmacy training, delivered the $\mathrm{AD}$ program to PCPs. The detailers were trained to deliver key messages from the CDC Guideline for Prescribing Opioids for Chronic Pain. ${ }^{24}$ Educational materials were created based on the CDC's guideline. Provider-specific opioid prescribing metrics were also provided at each visit and supplemental educational material available from the state health department. The aim of the AD program was to provide PCPs evidence-based information to impact their management of patients with chronic pain. Attempts to keep the same detailer across both visits were made to facilitate trust and relationship building.

After each visit, the detailers were asked to record their responses to the developed instrument through an online survey tool (ie, Google poll) within 30 minutes of visit completion. Responses were automatically uploaded to a secure remote server. The UIC Institutional Review Board approved this study, and written informed consent was obtained from all providers at the initial visit.

\section{Analysis}

A psychometric evaluation of the instrument was conducted using classical test theory (CTT) to assess internal consistency reliability, exploratory factor analysis (EFA) to identify the dimensionality of the measure, and item response theory (IRT) based methods to determine item fit and redundancy. These methods were applied to data from both the first and second visits to confirm results. Item nonresponse was reported to identify if any of the items had feasibility issues ( $\mathbf{5} \%$ nonresponse was considered acceptable). ${ }^{25}$ Floor and ceiling effects were evaluated, which refers to the tendency of the responses to an item to skew toward the lower or higher end of the scale, respectively. If items are worded negatively, their response scores are reverse coded before summation.

Reliability of the instrument was assessed to ensure the consistency of scale measurements. Internal consistency of the instrument was evaluated by Cronbach a ( $\searrow 0.70$ as minimally acceptable). ${ }^{23}$ Item-item correlation was also evaluated to determine the level of correlation between each item ( $\searrow 0.25$ as minimally acceptable) ${ }^{23}$ Corrected item-total score correlation 
$(\searrow 0.50 \text { as minimally acceptable })^{26}$ was used to evaluate item discrimination and how each item correlated with the entire scale. Correlations were evaluated using Spearman rank-order correlation coefficient (rho) because a Likert scale was used.

EFA was conducted to evaluate the number of common themes and groupings of the items within the instrument. The Kaiser-Meyer-Olkin (KMO) value was evaluated to confirm the data was suitable for EFA (KMO $\geq 0.7$ as acceptable). ${ }^{27,28}$ Various methods of EFA with different rotations were considered. A polychoric correlation matrix, principal axis factoring, with a varimax rotation was used. A polychoric correlation matrix was used because the data being analyzed were categorical by nature. ${ }^{29}$ Ultimately, principal component analysis was used because the data were not normally distributed. A varimax rotation method, which is a type of orthogonal rotation that assumes uncorrelated factors and maximizes the factor loadings, was also used to assess multiple factors in our data. ${ }^{26}$ Factor loadings and unidimensionality were examined at an Eigenvalue of 1.0, and a scree plot was generated for each visit.

A psychometric evaluation of the instrument was conducted using IRT. We used the graded response model that was fitted to the data because a Likert scale was used where the response options are ordered. Item information functions were used to illustrate the amount of information each item provided for estimating the latent trait (theta) of interest. Test information functions were generated to assess the amount of information for the set of items across ranges of theta. The test information at a given theta (ability) is the sum of the individual item information at that level of ability. The test information function is an indicator of how well a given set of items can estimate the trait of interest (theta) across different levels of ability. The standard error measurement for the test across different values of theta was also generated. A post-hoc analysis was conducted to refine the instrument.

\section{RESULTS}

\section{Instrument Development}

Five items were generated and presented to external content experts based on the themes identified in the literature review. After consultation, an additional item related to the theme of readiness to change was included (item 6). A six-item instrument was ultimately developed. Item three was worded negatively (ie, "it is not feasible...") for the first visit and was reworded for the second visit (ie, "it is feasible...") to address the potential concern that a negatively worded item would engender a different response pattern.

\section{Sample Population and Survey Administration}

Most of the clinicians visited were Doctors of Medicine (52.5\%), followed by Doctor of Osteopathic Medicine (33.3\%), nurse practitioners (9.8\%), and physician assistants (4.4\%) (Table 1). Most of the clinicians were women (55.2\%), nonresidents (83.1\%), with an average of 15 years in practice. There were 183 baseline visits with 158 follow-up visits. Providers lost to follow-up, most of whom were either Doctors of Medicines (48\%) and residents (48\%), were due to refusal, scheduling conflicts, clinic site closings, and resident 
turn over. The first visit averaged 14.3 minutes $(\mathrm{SD}=0.2)$, whereas the second visit averaged 11.6 minutes $(\mathrm{SD}=0.2)$.

\section{Psychometric Analysis}

One entire survey was missing from the initial provider visits (Table 2) resulting in 182 observations for analysis. There was a $100 \%$ response rate $(n=158)$ for the second visit. The mean scores for all items (except for item 3 related to feasibility) in both visits were positively skewed to the right as seen by the mean scores above 3 . More than $50 \%$ of responses were either reported as "very" or "extremely" for each item. Because item 3 (feasibility) was worded negatively, its response in visit 1 was reverse scored. The maximum possible score was 30 , whereas the minimum was 6 . The average total score was 22.9 ( $\mathrm{SD}=$ 3.4) for visit 1 and $22.8(\mathrm{SD}=3.5)$ for visit 2.

Item-item correlations for visits 1 and 2 can be seen in Table 3 . In visit 1 , item 3 (feasibility) correlated poorly with most of the other items (rho $<0.16$ ) except for item 2 (acceptability). In visit 2, only the correlation between items 3 (feasibility) and 4 (relevance) was below the minimum acceptable value $($ rho $=0.16)$. Items 1,2 , and 6 related to usefulness, acceptability, and readiness to change, respectively, were most highly correlated with each other for both visit 1 (rho $=0.52-0.70)$ and visit $2($ rho $=0.55-0.67)$. The corrected item-total correlations were all above the minimum acceptable level of rho $\searrow 0.5$ except for items 3,4 , and 5 (feasibility, relevance, and communication). Item 1, 2, and 6 (usefulness, acceptability, and readiness to change) resulted in the highest corrected item-total correlations for visit 1 (rho $=0.58-0.67)$ and visit $2(\mathrm{rho}=0.65-0.71)$. Cronbach $a$ for the measure was greater than 0.7 (visit $1=0.74$, visit $2=0.79$ ) for both visits which indicated an acceptable level of internal consistency. Cronbach $a$ increased after omitting item 3 related to feasibility $(a=0.80)$ which persisted even after rewording the item, suggesting that it is the least homogenous in the instrument.

KMO values for both visits were adequate (visit 1: 0.68 and visit 2:0.82) suggesting the data are acceptable for factor analysis. After rewording item 3 (feasibility) for visit 2, all items loaded onto one factor suggesting that all the items are now relevant in defining the factor's dimensionality (see Table 1, Supplemental Digital Content 1, http://links.lww.com/JCEHP/ A95). Even so, item 3 had the lowest factor loading (0.51) and most uniqueness (0.74) suggesting that it may be more appropriate to report it separately from the other items.

Item and test information functions for visits 1 are seen in Figure 1, which are very similar to those in visit 2 (see Figure 1, Supplemental Digital Content 2, http://links.lww.com/ JCEHP/A94). Items 1, 2, and 6 (usefulness, acceptability, and readiness to change) seemed to provide the most information across both visits, whereas items 3,4 , and 5 (feasibility, relevance, and communication effectiveness) consistently provided the least amount of information across the ranges of theta. When items 3, 4, and 5 are omitted, there is no significant change in the test information function for both visits. However, interestingly, the amount of information for items 1 (usefulness) and 6 (readiness to change) seem to invert when the other three items are removed. The most error and least information provided on the test information functions occurred at two ends of the scale suggesting that the instrument may be poor at differentiating among extremely high or low perceive AD visit 
effectiveness. Finally, the amount of information provided in visit 2 was lower than visit 1 , which may be due to the population lost to follow-up.

A post-hoc analysis was conducted to further explore the relationships among the items. Using a backward elimination process, when only items 1, 2, and 6 (usefulness, acceptability, and readiness to change) were evaluated together, the total Cronbach a increased $(a=0.83)$, and omitting each item subsequently decreased the Cronbach $a$ suggesting each of the three items contributed to the proposed scale's internal consistency. In addition, the corrected item-total correlations among the three items were all rho $>0.60$. These results suggest the items related to usefulness, acceptability, and readiness to change measured the latent trait the best, whereas the items related to feasibility (items 3 ) and communication (item 5) seemed to measure slightly different constructs.

\section{DISCUSSION}

A five-item instrument to measure the perceived effectiveness of an opioid-focused AD program was developed and psychometrically evaluated. Although the instrument was implemented in an $\mathrm{AD}$ program focused on appropriate opioid prescribing, it was intentionally developed to be generalizable across different therapeutic areas.

All items contained in the instrument loaded onto one factor after adjusting for negative wording in visit 2, suggesting the instrument is unidimensional. However, three items related to usefulness, acceptability, and readiness to change (items 1,2, and 6) were found to provide the most information based on CTT and IRT analysis. These three items seem to load together onto a single construct which we hypothesize to be a "likelihood to change" that contributes to perceived AD visit effectiveness and can be treated as a scale to derive a total summary score by adding their response scores together. We hypothesize that the items related to usefulness, acceptability, and readiness to change worked successively. For example, a provider is more willing to implement information that they find useful and, therefore, are more ready to change their practice behavior. Items related to feasibility (item 3 ) and communication effectiveness (item 5) should be reported separately from the aforementioned items based on IRT analysis. However, because these constructs are still important components to measure when evaluating an $\mathrm{AD}$ program, they are therefore included in the instrument. The authors propose a hypothesized model to provide a possible framework for how these items are working together.

The basis of dropping the item related to relevance (item 4) was informed by the Cronbach $a$ and IRT analysis. The lack of change in the Cronbach $a$ and slightly higher item-item correlations after omitting it in visit 2 suggest that it could be redundant to item 1 (Table 3). Based on the analysis, it is possible the word "relevant" in item 4 may be perceived as similar to the word "useful" in item 1. For example, if a detailer perceived the key message is relevant to the provider's practice, then it may be more likely to be perceived as useful rather than or merely being informative. Furthermore, dropping this item would decrease response burden as well. We acknowledge that the double-barreled wording of item 1 (usefulness) is problematic (ie, "informative/useful"), and we justify removing item 4 (relevance) rather than item 1 because we believe the key element to perceived effectiveness 
and to promoting clinician change in behavior is the usefulness of the visit. Furthermore, we justify dropping the word "informative" in item 1 because, again, the key element to assessing perceived $\mathrm{AD}$ visit effectiveness and changing clinician behavior is usefulness rather than merely being informative.

Several limitations are worth noting. This study is the first step toward the accruement of evidence on the validity of this measure of detailer perception of AD effectiveness. The external aspects of the instrument's construct validity with other related measures, such as the prescriber satisfaction with academic detailing,${ }^{30}$ another measure under development, represents future research opportunities. Furthermore, the instrument's ability to predict changes in opioid prescribing will be evaluated after obtaining opioid prescribing data from the Illinois Prescription Monitoring Program. Properties that warrant further investigation include responsiveness of the instrument and test-retest reliability because the current study was not designed to evaluate the stability of the measure. As mentioned above, the doublebarreled wording of item was problematic and may have led to respondent confusion. Future testing should remove the word "informative" based on reasoning stated above. Future research will also evaluate response differences because of random ordering of items, which will require larger sample sizes to detect any differences.

The strengths of this study include the psychometric analysis of a novel instrument developed to measure the perceived effectiveness of an AD program using CTT and IRT methods. To the best of our knowledge, this is the first psychometrically validated instrument of perceived AD effectiveness by the detailer to be used in an AD program. This instrument was designed for generic use in $\mathrm{AD}$ programs, regardless of the disease or therapeutic backgrounds because the conceptual domains are relevant to all AD program effectiveness. Spearman's correlation coefficient provided a more conservative evaluation of correlation values given the response scale of the instrument (ie, Likert scale). The corrected item-total correlations satisfied the minimal requirement deemed acceptable. The instrument also demonstrated high levels of internal consistency.

\section{CONCLUSION}

We developed a novel five-item detailer assessment of visit effectiveness (the "DAVE") instrument to evaluate the perceived effectiveness of an AD program focused on safe and appropriate opioid prescribing. Initial evidence supports the validity and reliability of the measure, which can be further supported by evaluating the predictive ability of the instrument for actual changes in prescribing behavior. Further study of the relationship of items using structural equation modeling will assist in validating the constructs as conceptualized. In addition, further insight into the validity and generalizability of the measure through application in other AD studies is recommended.

\section{Supplementary Material}

Refer to Web version on PubMed Central for supplementary material. 


\section{ACKNOWLEDGMENTS}

Academic Detailers from University of Illinois at Chicago College of Pharmacy: Victoria Kulbokas, Esther Lee, Shannon Menard, Ammarah Nadeem, Dayna Redini, Aleksandra Ruseva, Sarette Tilton, and Nevena Varagic. AMITA Health: Darin Jordan, MD; Ankur Dave, MD; and Reinhold Llerena, MD. Illinois Prescription Monitoring Program: Craig Berberet; Stan Murzynski; Ed Dowllar; Sarah Pointer, PharmD; and Andrew Hollo. Expert panel: David Liebowitz, MD; Scott Glaser, MD; Chris Herndon, PharmD; and Julie Adkins DNP.

Disclosure: M. A. Fischer is a clinical consultant for Alosa Health, a health education nonprofit that provides academic detailing services. The remaining authors declare no conflict of interest. The investigators and project were supported by the following: (1) Centers for Disease Control and Prevention: Prevention for States (PfS) by the Illinois Department of Human Services and Illinois Prescription Monitoring Program, Grant\#: 49I1099M40. and (2) Centers for Disease Control and Prevention of the U.S. Department of Health and Human Services (HHS), Grant \# R01 CE003156, as part of a financial assistance award totaling \$1,537,532 to date with 25 percent funded by CDC/ HHS. The contents are those of the author(s) and do not necessarily represent the official views of, nor an endorsement, by CDC/HHS, or the U.S. Government. Approval obtained by the University of Illinois at Chicago Institutional Review Board.

\section{REFERENCES}

1. Scholl L, Seth P, Kariisa M, et al. Drug and opioid-involved overdose deaths-United States, 2013-2017. MMWR Morb Mortal Wkly Rep. 2018;67:1419-1427. [PubMed: 30605448]

2. Levy B, Paulozzi L, Mack KA, et al. Trends in opioid analgesic-prescribing rates by specialty, U.S., 2007-2012. Am J Prev Med. 2015;49:409-13. [PubMed: 25896191]

3. Guy GP Jr, Zhang K. Opioid prescribing by specialty and volume in the U.S. Am J Prev Med. 2018;55:e153-e155. [PubMed: 30219212]

4. Jamison RN, Sheehan KA, Scanlan E, et al. Beliefs and attitudes about opioid prescribing and chronic pain management: survey of primary care providers. J Opioid Manag. 2014;10:375-382. [PubMed: 25531955]

5. Mezei L, Murinson BB; Johns Hopkins Pain Curriculum Development T. Pain education in North American medical schools. J Pain. 2011;12:1199-1208. [PubMed: 21945594]

6. Keller CE, Ashrafioun L, Neumann AM, et al. Practices, perceptions, and concerns of primary care physicians about opioid dependence associated with the treatment of chronic pain. Subst Abus. 2012;33:103-113. [PubMed: 22489582]

7. Pearson AC, Moman RN, Moeschler SM, et al. Provider confidence in opioid prescribing and chronic pain management: results of the Opioid Therapy Provider Survey. J Pain Res. 2017;10:1395-1400. [PubMed: 28652805]

8. Upshur CC, Luckmann RS, Savageau JA. Primary care provider concerns about management of chronic pain in community clinic populations. J Gen Intern Med. 2006;21:652-655. [PubMed: 16808752]

9. Soumerai SB, Avorn J. Principles of educational outreach ("academic detailing") to improve clinical decision making. JAMA. 1990;263:549-556. [PubMed: 2104640]

10. O'Brien MA, Rogers S, Jamtvedt G, et al. Educational outreach visits: effects on professional practice and health care outcomes. Cochrane Database Syst Rev. 2007:CD000409. [PubMed: 17943742]

11. Yeh JS, Van Hoof TJ, Fischer MA. Key features of academic detailing: development of an expert consensus using the Delphi method. Am Health Drug Benefits. 2016;9:42-50. [PubMed: 27066195]

12. Proctor E, Silmere H, Raghavan R, et al. Outcomes for implementation research: conceptual distinctions, measurement challenges, and research agenda. Adm Policy Ment Health. 2011;38:6576. [PubMed: 20957426]

13. Gitlow HS, Melby MJ. Framework for continuous quality improvement in the provision of pharmaceutical care. Am J Hosp Pharm. 1991;48:1917-1925. [PubMed: 1928133]

14. Kattan JA, Tuazon E, Paone D, et al. Public health detailing-A successful strategy to promote judicious opioid analgesic prescribing. Am J Public Health. 2016;106:1430-1438. [PubMed: 27400353] 
15. Liebschutz JM, Xuan Z, Shanahan CW, et al. Improving adherence to long-term opioid therapy guidelines to reduce opioid misuse in primary care: a cluster-randomized clinical trial. JAMA Intern Med. 2017;177:1265-1272. [PubMed: 28715535]

16. Oliva EM, Christopher MLD, Wells D, et al. Opioid overdose education and naloxone distribution: development of the Veterans Health Administration's national program. J Am Pharm Assoc (2003). 2017;57:S168-S179 e164. [PubMed: 28292502]

17. Cochella S, Bateman K. Provider detailing: an intervention to decrease prescription opioid deaths in Utah. Pain Med. 2011;12(suppl 2):S73-S76. [PubMed: 21668760]

18. Larson MJ, Browne CP, Nikitin RV, et al. Physicians report adopting safer opioid prescribing behaviors after academic detailing intervention. Subst Abus. 2018;39:218-224. [PubMed: 29608412]

19. Voelker KA, Schauberger C. Academic detailing for postpartum opioid prescribing. J Am Board Fam Med. 2018;31:944-946. [PubMed: 30413551]

20. Abd-Elsayed A, Albert CA, Fischer M, et al. Naloxone academic detailing: role of community outreach teaching. Curr Pain Headache Rep. 2018;22:72. [PubMed: 30151695]

21. Van Hoof TJ, Harrison LG, Miller NE, et al. Characteristics of academic detailing: results of a literature review. Am Health Drug Benefits. 2015;8:414-422. [PubMed: 26702333]

22. Anthierens S, Verhoeven V, Schmitz O, et al. Academic detailers' and general practitioners' views and experiences of their academic detailing visits to improve the quality of analgesic use: process evaluation alongside a pragmatic cluster randomized controlled trial. BMC Health Serv Res. 2017;17:841. [PubMed: 29268730]

23. Streiner DL, Norman GR. Health Measurement Scales: A Practical Guide to Their Development and Use. 4th ed. Oxford, United Kingdom; New York, NY: Oxford University Press; 2008.

24. Dowell D, Haegerich TM, Chou R. CDC guideline for prescribing opioids for chronic painUnited States, 2016. MMWR Recomm Rep. 2016;65:1-9.

25. Petrillo J, Cano SJ, McLeod LD, et al. Using classical test theory, item response theory, and Rasch measurement theory to evaluate patient-reported outcome measures: a comparison of worked examples. Value Health. 2015;18:25-34. [PubMed: 25595231]

26. Nunnally JC, Bernstein IH. Psychometric Theory. 3rd ed. New York, NY: McGraw-Hill; 1994.

27. Box GE. A general distribution theory for a class of likelihood criteria. Biometrika. 1949;36:317346. [PubMed: 15402070]

28. Cureton EE, D’Agostino RB. Factor Analysis, an Applied Approach. Hillsdale, NJ: L. Erlbaum Associates; 1983.

29. Lee SY, Leung KM. Estimation of multivariate polychoric and polyserial correlations with missing observations. Br J Math Stat Psychol. 1992;45(pt 2):225-238. [PubMed: 1472450]

30. Monteiro A, Smart M, Saffore C, et al. Development of a measure of prescriber satisfaction with academic detailing. Value in Health. 2019;22:S260.

J Contin Educ Health Prof. Author manuscript; available in PMC 2021 October 01. 


\section{Lessons for Practice}

- $\quad$ Perceived effectiveness of an AD visit can be evaluated, especially in lieu of direct feedback from providers.

- The instrument is designed to be generalizable across different AD programs.

- $\quad$ The instrument can serve as a formative evaluation tool of $\mathrm{AD}$ programs across multiple visits. 

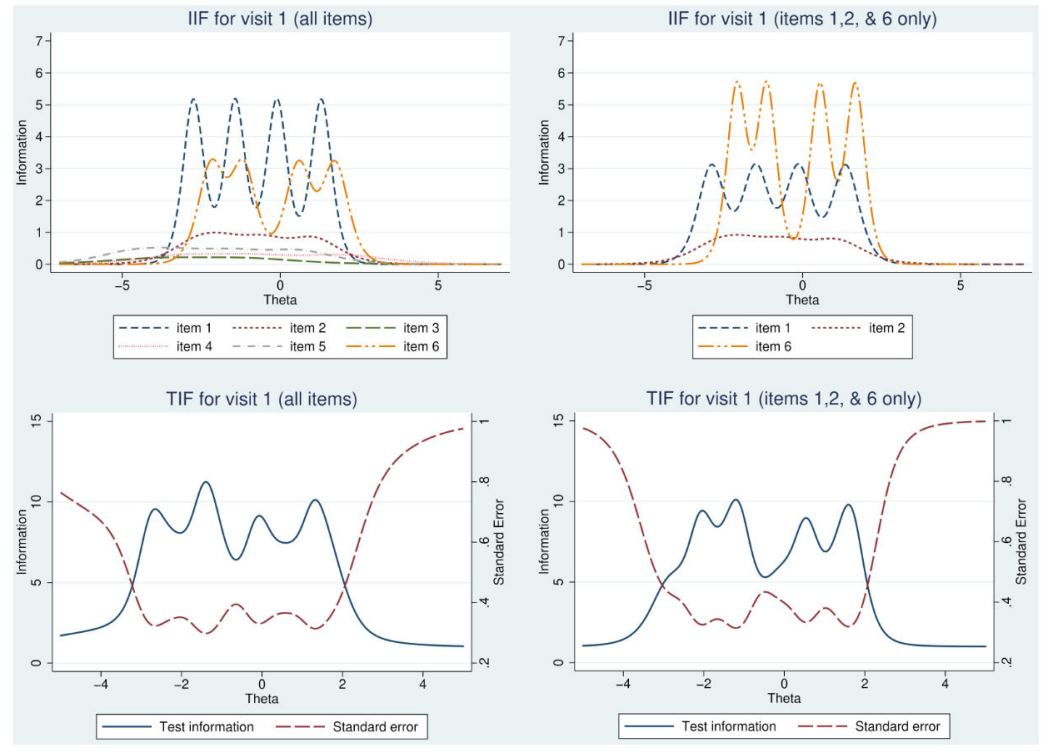

FIGURE 1.

Item information function and test information function graphs for visit 1 for all items (items 1-6) and selected items (items 1, 2, and 6). IIF, item information function, TIF, test information function. 

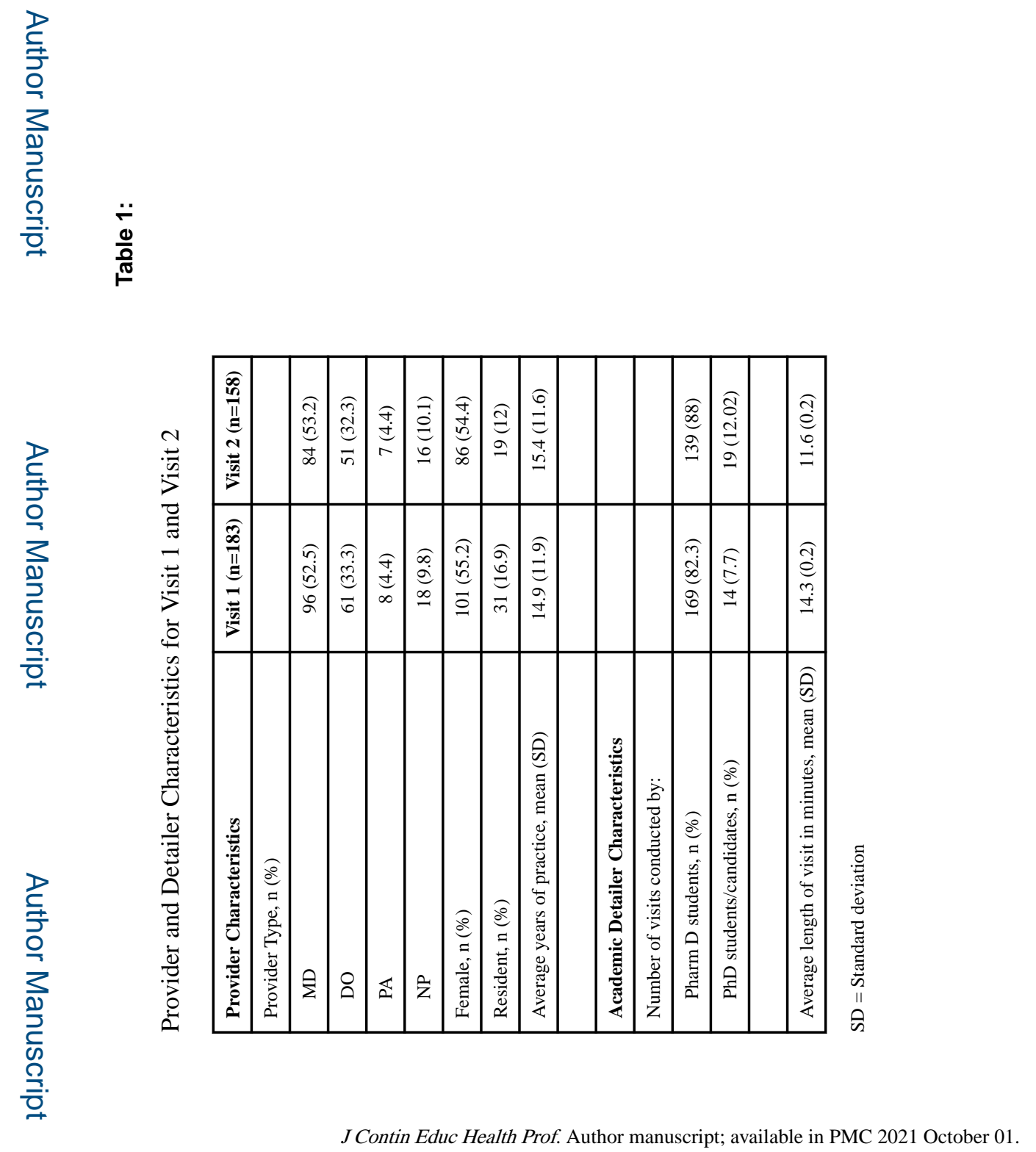


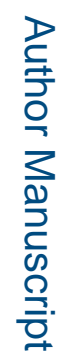

\begin{tabular}{|c|c|c|c|c|c|c|c|c|c|c|c|c|c|}
\hline 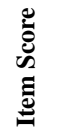 & 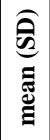 & 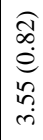 & $\mid \begin{array}{c}\widehat{a} \\
\hat{d} \\
\hat{\sigma} \\
\hat{n} \\
\dot{n}\end{array}$ & 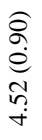 & 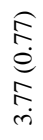 & 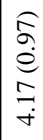 & 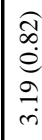 & $\begin{array}{l}\widehat{\partial} \\
\stackrel{0}{0} \\
\stackrel{0}{0} \\
\dot{c}\end{array}$ & 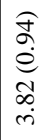 & $\begin{array}{l}\widehat{\infty} \\
\stackrel{0}{e} \\
\stackrel{0}{0} \\
\dot{+} \\
\dot{+}\end{array}$ & 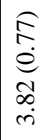 & 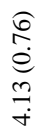 & $\begin{array}{c}\approx \\
\infty \\
e \\
m \\
m \\
\end{array}$ \\
\hline 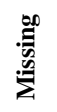 & $\underset{=}{\stackrel{d}{e}}$ & 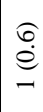 & $\mid \begin{array}{c}0 \\
\stackrel{0}{0} \\
-\end{array}$ & 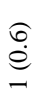 & 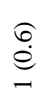 & 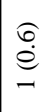 & $\stackrel{6}{\stackrel{6}{e}}$ & $\hat{\varrho}$ & $\widehat{\varrho}$ & @o & $\hat{\varrho}$ & $\hat{\varrho}$ & $\hat{\varrho}$ \\
\hline 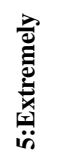 & 8 & 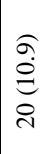 & $\left|\begin{array}{c}\hat{\mathcal{d}} \\
\vec{d} \\
\mathcal{\gamma}\end{array}\right|$ & 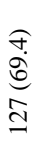 & 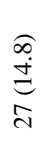 & 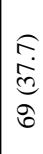 & 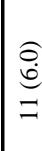 & 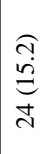 & 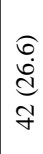 & $\mid \begin{array}{l}\hat{\tau} \\
\tilde{d} \\
\tilde{g} \\
\tilde{\gamma}\end{array}$ & $\begin{array}{l}\hat{\sigma} \\
\stackrel{n}{ٍ} \\
\stackrel{d}{d}\end{array}$ & 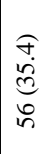 & 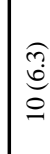 \\
\hline
\end{tabular}

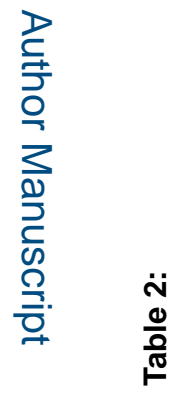

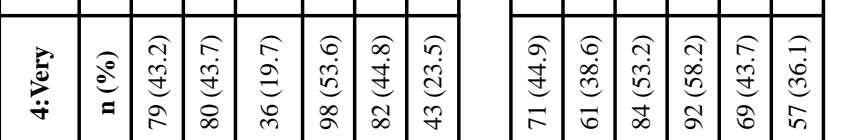

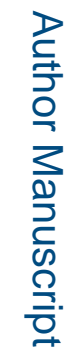

\begin{tabular}{|c|c|c|c|c|c|c|c|c|c|c|c|c|c|}
\hline 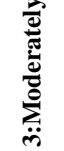 & $\stackrel{0}{=}$ & 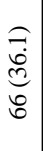 & $\mid \begin{array}{c}\tilde{n} \\
\tilde{d} \\
\tilde{d} \\
\tilde{q}\end{array}$ & $\begin{array}{l}\hat{\sigma} \\
\text { ḋ } \\
\text { a }\end{array}$ & $\begin{array}{l}\text { 今. } \\
\text { d. } \\
\text { ơ }\end{array}$ & 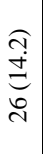 & 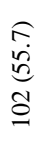 & $\begin{array}{l}\widehat{\infty} \\
\dot{0} \\
\dot{d} \\
i n \\
i n\end{array}$ & 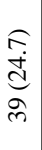 & 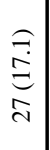 & $\begin{array}{l}6 \\
\dot{0} \\
\vec{E} \\
\vec{m}\end{array}$ & 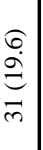 & $\begin{array}{l}\underset{\partial}{\dot{\gamma}} \\
\underset{\infty}{0}\end{array}$ \\
\hline 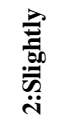 & $\stackrel{Ð 0}{=}$ & 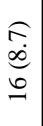 & $\mid \begin{array}{l}n \\
0 \\
0 \\
0\end{array}$ & $\begin{array}{l}\hat{\jmath} \\
\stackrel{్}{6}\end{array}$ & $\begin{array}{l}\hat{\theta} \\
\stackrel{\theta}{=} \\
=\end{array}$ & $\begin{array}{l}\underset{\widehat{T}}{\mathrm{~d}} \\
\vec{\sigma}\end{array}$ & $\begin{array}{l}\stackrel{n}{\Xi} \\
\underset{\sim}{\Xi}\end{array}$ & $\underset{r}{\stackrel{f}{+}}$ & $\begin{array}{l}\widehat{\overbrace{}} \\
\stackrel{0}{0} \\
\underline{6}\end{array}$ & 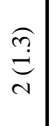 & 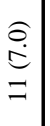 & $\underset{\sim}{\stackrel{\widehat{?}}{\rightleftarrows}}$ & $\begin{array}{l}\stackrel{\widehat{m}}{\vec{g}} \\
\underset{\vec{v}}{2}\end{array}$ \\
\hline 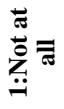 & $\underset{=}{\stackrel{0}{e}}$ & 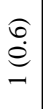 & $\mid \begin{array}{c}\widehat{\alpha} \\
\stackrel{0}{\sim} \\
\therefore\end{array}$ & $\underset{\vec{J}}{\stackrel{d}{d}}$ & $\hat{\varrho}$ & $\begin{array}{l}\stackrel{6}{e} \\
\stackrel{e}{-}\end{array}$ & $\begin{array}{l}\hat{d} \\
i\end{array}$ & $\stackrel{6}{\stackrel{6}{\Theta}}$ & $\stackrel{\varrho}{\varrho}$ & 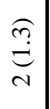 & $\hat{\varrho}$ & @़े & $\underset{\sim}{\stackrel{\widehat{\vartheta}}{\rightleftarrows}}$ \\
\hline
\end{tabular}



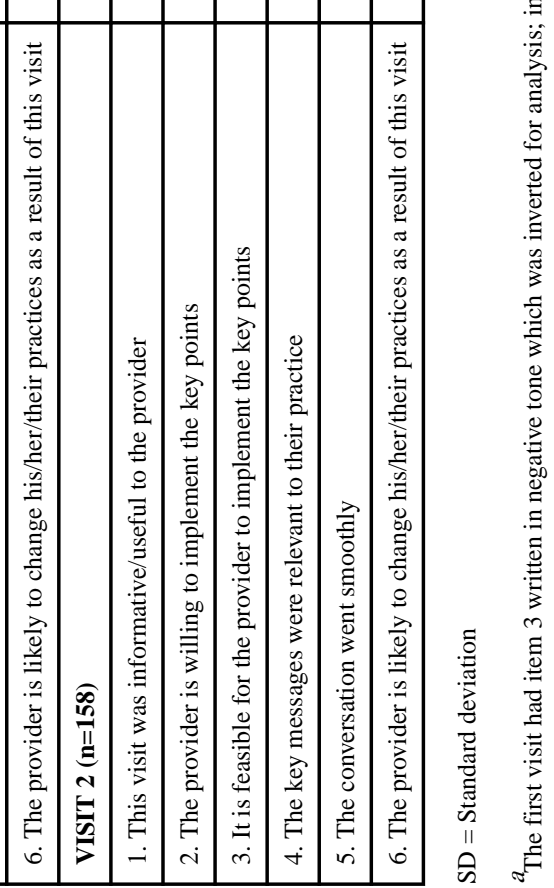

J Contin Educ Health Prof. Author manuscript; available in PMC 2021 October 01. 


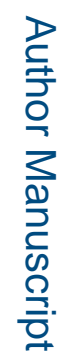

로을

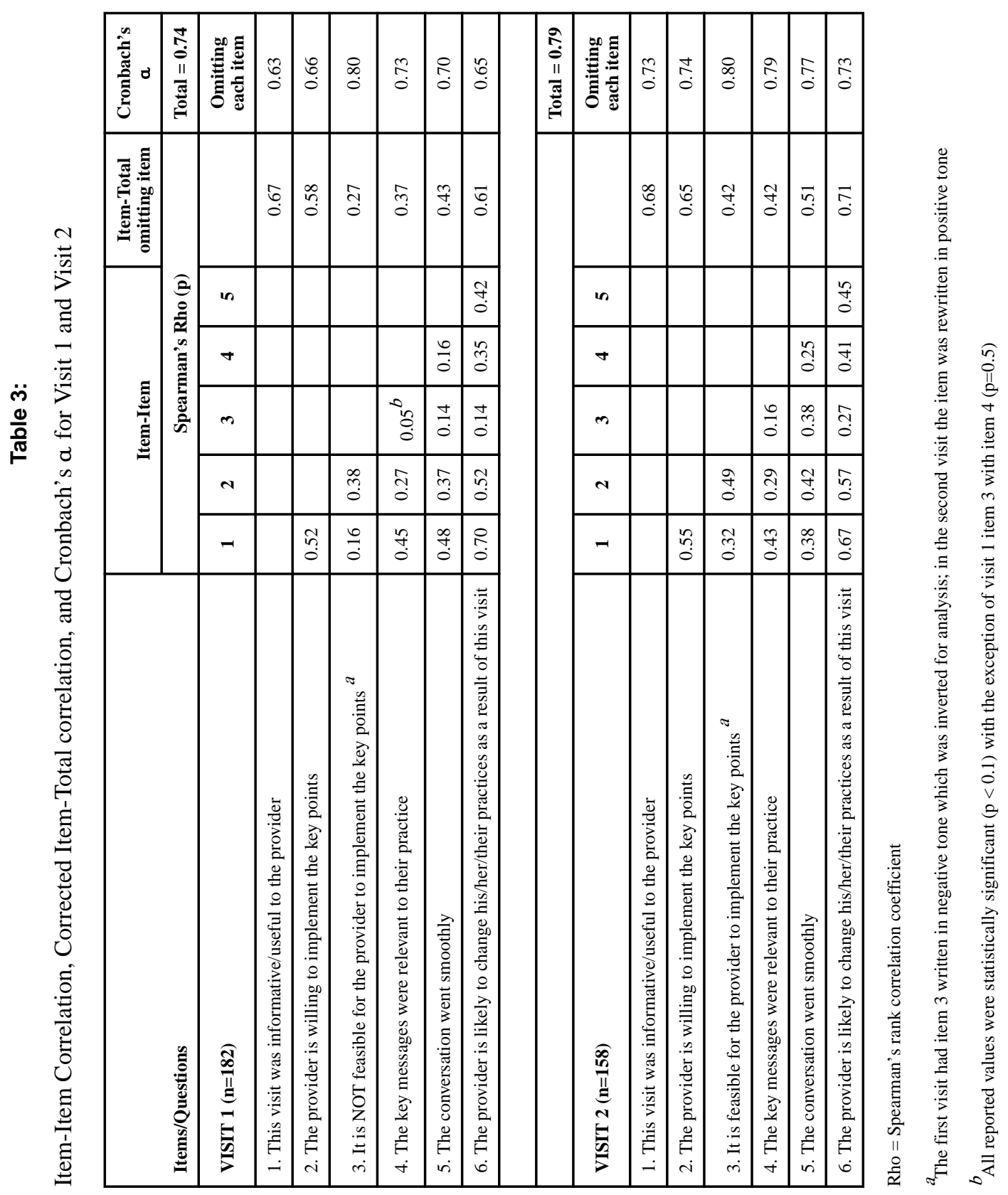

J Contin Educ Health Prof. Author manuscript; available in PMC 2021 October 01. 